\title{
Stick-slip and Torsional Friction Factors in Inclined Wellbores
}

\author{
Ulf Jakob F. Aarsnes ${ }^{1,2, \star}$ and Roman J. Shor ${ }^{3, \star \star}$ \\ ${ }^{1}$ International Research Institute of Stavanger (IRIS), Oslo, Norway \\ ${ }^{2}$ DrillWell - Drilling and well centre for improved recovery, Stavanger, Norway \\ ${ }^{3}$ University of Calgary, Department of Chemical and Petroleum Engineering, Calgary, Canada
}

\begin{abstract}
Stick slip is usually considered a phenomenon of bit-rock interaction, but is also often observed in the field with the bit off bottom. In this paper we present a distributed model of a drill string with an alongstring Coulomb stiction to investigate the effect of borehole inclination and borehole friction on the incidence of stick-slip. This model is validated with high frequency surface and downhole data and then used to estimate static and dynamic friction.

A derivation of the torsional drill string model is shown and includes the along-string Coulomb stiction of the borehole acting on the string and the 'velocity weakening' between static and dynamic friction. The relative effects of these two frictions is investigated and the resulting drillstring behavior is presented. To isolate the effect of the along-string friction from the bit-rock interaction, field data from rotational start-ups after a connection (with bit off bottom) is considered. This high frequency surface and downhole data is then used to validate the surface and downhole behavior predicted by the model.

The model is shown to have a good match with the surface and downhole behavior of two deviated wellbores for depths ranging from 1500 to 3000 meters. In particular, the model replicates the amplitude and period of the oscillations, in both the topside torque and the downhole RPM, as caused by the along-string stick slip. It is further shown that by using the surface behavior of the drill-string during rotational startup, an estimate of the static and dynamic friction factors along the wellbore can be obtained, even during stick-slip oscillations, if axial tension in the drillstring is considered. This presents a possible method to estimate friction factors in the field when off-bottom stick slip is encountered, and points in the direction of avoiding stick slip through the design of an appropriate torsional start-up procedure without the need of an explicit friction test.
\end{abstract}

\section{Introduction}

Exploration and production of oil and gas in the deep subsurface, where hydrocarbon reservoirs are found at depths between 2,000 and 36,000 feet [1], requires that a narrow borehole, between 4 and 24 inches in diameter, be drilled using a slender drill-string through a varied downhole environment and along an often snaking wellpath. Drill string vibrations, and their negative consequences on ROP and equipment, are a well known phenomenon when drilling for hydrocarbons. In particular, the torsional oscillations known as stick slip, which are considered to be some of the most prevalent vibrations, are to be avoided. These stick-slip oscillations are characterized by a series of stopping - "sticking" - and releasing - "slipping" events of the bit.

Significant literature exists which seeks to explain the incidence of stick slip through various implementations of bit-rock interaction and various complexities of drillstring dynamic models, summarized in [2]. Many models considers the drill-string as a lumped mass, representing the bottom hole assembly (BHA) inertia, and a torsional

\footnotetext{
^e-mail: ujfa@iris.no

${ }^{\star}$ e-mail: roman.shor@ucalgary.ca
}

spring, representing the drill-string stiffness [3, 4], while more realistic, higher order, models have also been considered [5-7]. Stick-slip is then introduced as a self-excited vibration (see example in Fig. 1, right hand side) by inducing instability through a bit-rock interaction as a velocity weakening frictional force (Stribeck-like effect) [8-10], or through a regenerative effect [11-13] causing an apparent velocity weakening effect.

A key factor in common with the above referenced literature is that the bit-rock interaction is used to explain the cause of stick slip. A consequence of this is that the models are unable to explain the occurrence of stick-slip with the bit off bottom (where there is no bit-rock interaction). Off-bottom stick slip is a well known phenomenon from the field, and when mentioned in literature is hypothesized to be caused by a negative difference between static and dynamic along-string Coulomb-type friction [14-16]. This is an important phenomena as it indicates that nonlinear frictional forces along the drill-string (and not just the bit rock interaction), in deviated or horizontal wells, plays a significant role in the torsional oscillatory behavior of drill-strings. This is also a qualitatively different mechanism for causing stick slip from the self-excited type 

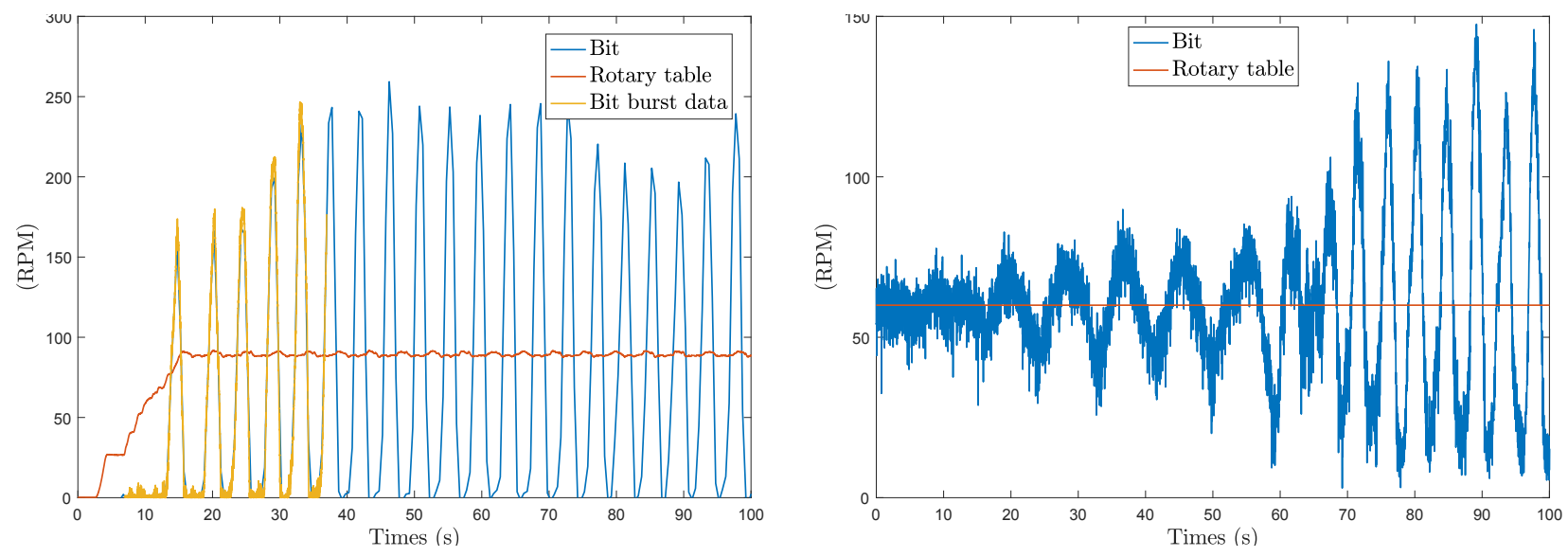

Figure 1: Two different kinds of stick-slip from two different wells. In the present paper we consider the type shown left, and not the self-excited type shown right.

caused by a velocity weakening effect in the bit-rock interaction, as is exemplified in Fig. 1:

- Self-excited vibrations are can occur when a slight change in e.g. bit-rock parameters causes the system to bifurcate and the resulting instability causes growing torsional oscillations to result in a stick-slip limit cycle. Shown right in Fig. 1.

- Off-bottom stick slip is cause by an initial exogenous shock, such as a rotation start-up, which pushes the drill string into activating the static Coulomb friction in the along string pipe-wellbore interaction. Shown left in Fig. 1.

Hence, models which only incorporate the bit rock interaction as the cause of torsional stick slip fail to explain off-bottom stick slip vibrations, as observed in field data after connections and in back-reaming operations. This potential cause of stick-slip, have not received nearly the same analysis or attention in the literature, in particular in the context of occurrence, characterization and avoidance.

\section{Model}

In this section we derive the model that will be used to recreate and analyze the torsional vibrations. To facilitate its relative simplicity, the formulation requires certain approximations, the main assumptions used are the following

- The bit is assumed to be off bottom and no bit-rock interaction is modeled.

- The torsional motion of the drill string is the dominating dynamic. No lateral or axial motion is assumed.

- The transition from static to dynamic Coulomb friction is modeled as a jump, i.e., the Stribeck curve is assumed negligible.

- The effects of along-string cuttings distribution on the friction is assumed to be homogenous.

- The effect of the pressure differential, inside and outside the drill string, on the bending moment is not represented and is assumed to be negligible.

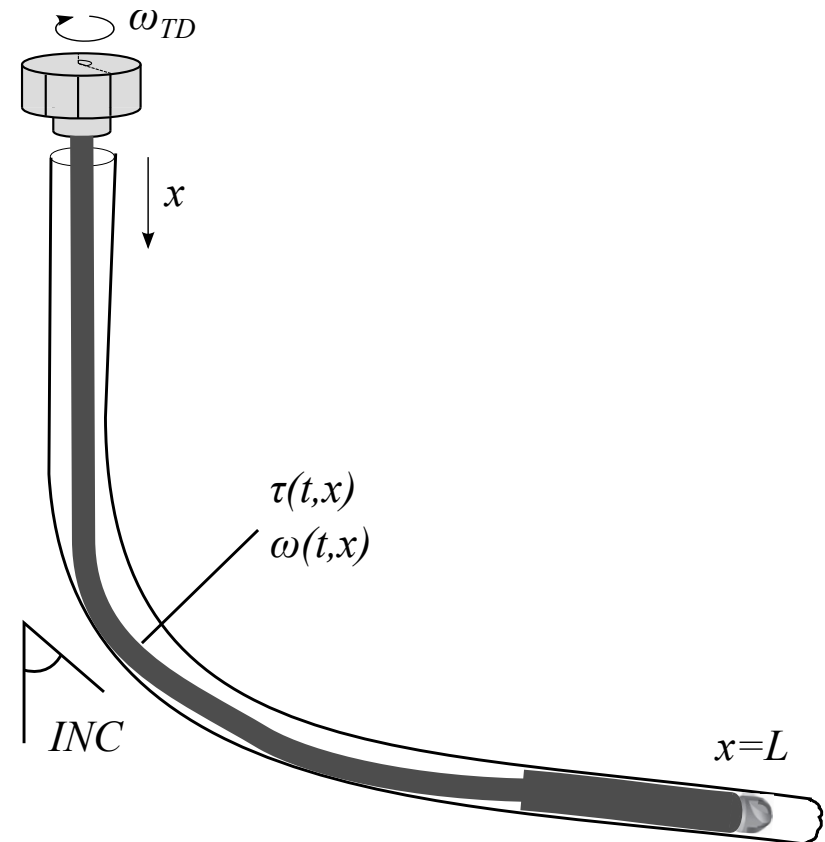

Figure 2: Schematic indicating the distributed drill string lying in deviate borehole.

\subsection{Torsional dynamics of the drill string}

We use a distributed model, similar to [17-19] and described in detail in [20], where we consider only the torsional dynamics of the drill string. For angular motion, angular velocity and torque are denoted as as $\omega(t, x), \tau(t, x)$, respectively, with $(t, x) \in[0, \infty) \times[0, L]$. See Fig. 2 for a schematic indicating locations. For an infinitesimal element $d x$, as shown in Fig. 3, the torque is found as the shear strain, or twist per unit length. Letting $\phi$ denote the angular displacement in the string s.t. $\frac{\partial \phi(t, x)}{\partial t}=\omega(t, x)$, we have $\tau(t, x)=J G(\phi(t, x)-\phi(t, x+d x)) / d x$, where $J$ is the polar moment for inertia and $G$ is the shear modulus. 

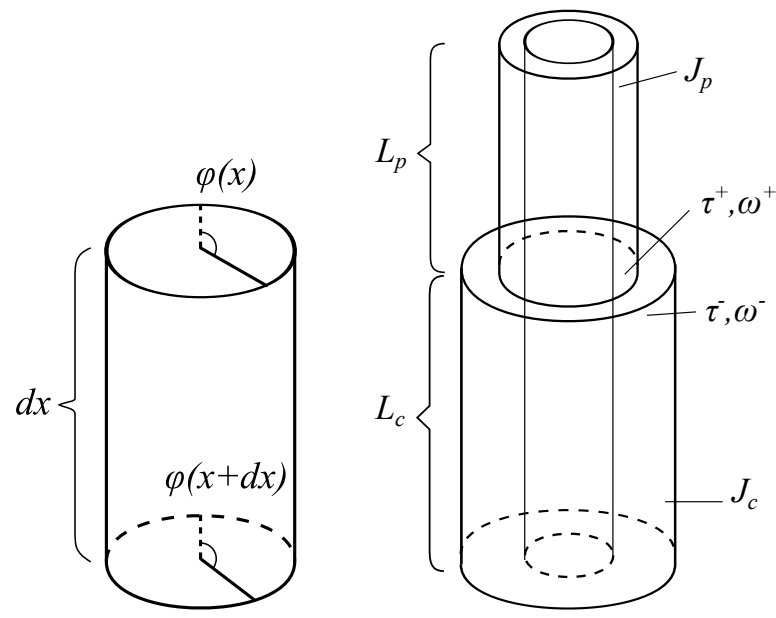

Figure 3: Infinitesmal drill string element (left) and the collar-Pipe transition (right).

Hence the equations for the angular motion are given by

$$
\begin{aligned}
& \frac{\partial \tau(t, x)}{\partial t}+J G \frac{\partial \omega(t, x)}{\partial x}=0 \\
& J \rho \frac{\partial \omega(t, x)}{\partial t}+\frac{\partial \tau(t, x)}{\partial x}=S(\omega, x),
\end{aligned}
$$

where the source term $S$ is modeled as

$$
S(\omega, x)=-k_{t} \rho J \omega(t, x)-\mathcal{F}(\omega, x)
$$

where $k_{t}$ is a damping constant representing the viscous shear stresses and $\mathcal{F}(\omega)$ is a differential inclusion, to be described, representing the Coulomb friction between the drill string and the borehole. The viscous shear stress coefficient $k_{t}$ represnts the combined damping effects of the viscous shear of the drilling mud and the rolling contact between drill string and the cuttings bed.

The lowermost section of the drill string is typically made up of drill collars which may have a great impact on the drill string dynamic due to their added inertia. In particular, the transition from the pipes to collars in the drill string will cause reflections in the traveling waves due to the change in characteristic line impedance [17].

We split the drill string into a pipe section with polar moment of inertia and lengths $J_{p}, L_{p}$ and a collar section with the same parameters given as $J_{c}, L_{c}$. We use $\tau^{-}, \omega^{-}$to denote the strain and velocity at the top of the drill collar and $\tau^{+}, \omega^{+}$at the bottom of the pipe, see Fig. 3. Enforcing the boundary conditions $\omega^{+}=\omega^{-}$and $\tau^{+}=-\tau^{-}$at the transition.

\subsection{Coulomb friction as an inclusion}

The Coulomb friction is modeled as an inclusion

$$
\begin{cases}\mathcal{F}(\omega, x)=F_{d}(x), & \omega>\omega_{c}, \\ \mathcal{F}(\omega, x) \in\left[-F_{c}(x), F_{c}(x)\right], & |\omega|<\omega_{c}, \\ \mathcal{F}(\omega, x)=-F_{d}(x), & \omega<-\omega_{c},\end{cases}
$$

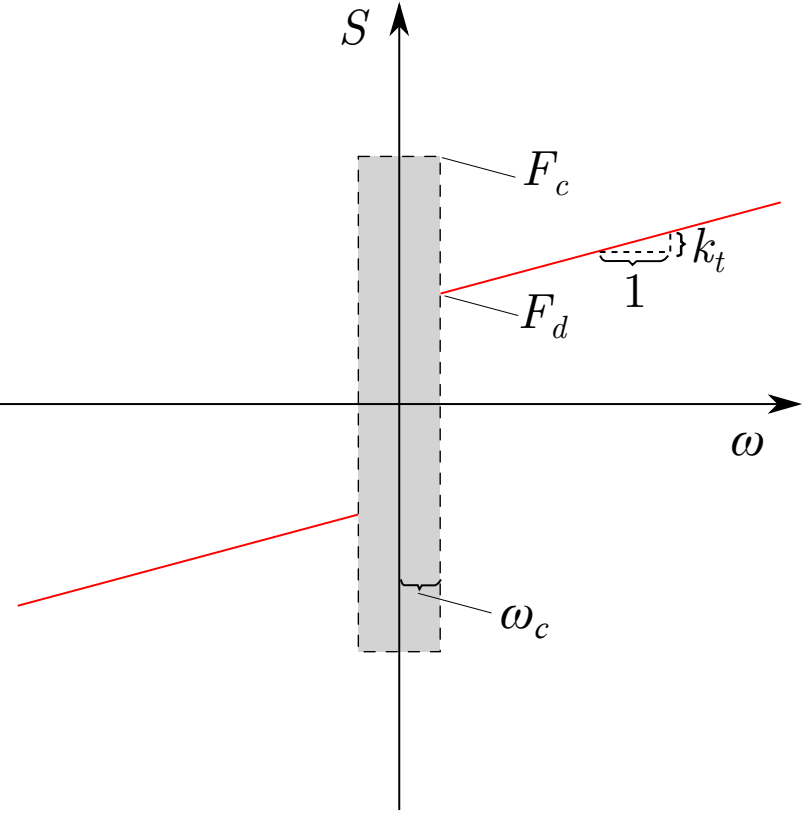

Figure 4: Schematic illustrating the four parameters determining the friction: the coulomb friction parameters $\omega_{c}, F_{c}, F_{d}$ and the viscous friction coefficient $k_{t}$, with the shaded region indicating the region of static torque, and the red curve the dynamic torque.

where $\omega_{c}$ is the threshold on the angular velocity where the Coulomb friction transitions from static to dynamic, $F_{d}$ is the dynamic Coulomb torque, $F_{c}$ is the static Coulomb torque, and $\mathcal{F}(\omega) \in\left[-F_{c}, F_{c}\right]$ denotes the inclusion where

$$
\mathcal{F}(\omega, x)=-\frac{\partial \tau(t, x)}{\partial x}-k_{t} \rho J \omega(t, x) \in\left[-F_{c}(x), F_{c}(x)\right],
$$

and take the boundary values $\pm F_{c}(x)$ if this relation does not hold. We define the non-dimensional coefficient $f_{\text {rat }}=$ $F_{c} / F_{d}$ to help characterize the magnitude of the oscillations. The shape of the friction source term is illustrated in Fig. 4.

Using the torque model of [21], we first derive the profile of the normal force $F_{N}(x)$ which is then used to express the torque.

The normal force is expressed in terms of the tension profile $\sigma_{e}$, assuming a planar well and torsional rotation of the drill string, the tension gradient is given as [21]:

$$
\frac{\partial \sigma_{e}}{\partial x}=W_{b} \cos \theta,
$$

where $W_{b}(x)=g A(x)\left(\rho-\rho_{\text {mud }}\right)$ is the buoyed weight per meter. That is, we have

$$
\sigma_{e}(x)=\int_{L}^{x} W_{b} \cos \theta(\xi) \mathrm{d} \xi
$$

The normal force profile, $F_{N}$, is then found as

$$
F_{N}(x)=\left(\sigma_{e}(x) \frac{\partial \theta}{\partial x}+W_{b} \sin (\theta)\right)
$$


and the maximum Coulomb torque

$$
F_{c}(x, \mu)=\mu r_{o}(x) F_{N}(x),
$$

where $\mu$ is the static Cuolomb friction factor. This friction factor is determined by factors such as wether the section is cased or openhole, as well as the lithology of the surrounding formation [14].

\subsection{Boundary condition}

At the topside boundary, the top drive is actuated by a motor torque, $\tau_{m}$, controlled by a PI controller to a desired velocity set-point $\omega_{S P}$ :

$$
\begin{aligned}
e & =\omega_{S P}-\omega_{T D} \\
I_{e} & =\int_{0}^{t} e(\xi) \mathrm{d} \xi \\
\tau_{m} & =k_{p} e+k_{i} I_{e},
\end{aligned}
$$

with a proportional gain, $k_{p}$ and an integral gain, $k_{i}$.

The topdrive has the inertia $J_{T D}$ and hence satisfies the dynamics

$$
\frac{\partial \omega_{T D}}{\partial t}=\frac{1}{J_{T D}}\left(\tau_{m}-\tau_{0}\right),
$$

and finally, the angular velocity at the top of the drill string is given as $\omega_{0}=\omega_{T D}$.

\subsubsection{Characterizing oscillations}

The period of the oscillations is a combination of the time required to build the twist needed to overcome the static Coulomb friction, and the torsional fundamental period of the drill string.

The required change in twist at the top-drive, $\Delta \phi(0)$, see [20] for details, enables us to find the build-up time of the cycle as

$$
t_{b u}=\frac{\Delta \phi(0)}{\omega_{T D}} .
$$

When the drill string is free, i.e. there is no build up time, the drill string oscillation will be dominated by the fundamental period of the free drill string, which is dependent on the drill pipe and collar length and the relative impedance $\bar{Z}$, [18]. Similarly, as the drill string breaks free, the trajectory will follow close to one fundamental period before getting stuck again.

That is, denoting the fundamental period of the drill string as $t_{f p}$, the period of the limit cycle is

$$
t_{p}=t_{b u}+t_{f p} .
$$

This allows us to heuristically distinguish between two different types of oscillations:

1. The oscillation is dominate by the fundamental period of the drill string: $t_{b u}<t_{f p}$. The torque trend resembles that of free oscillations.

2. The oscillation is dominated by the torque build up. A characteristic sawtooth pattern of the torque is obtained: $t_{b u}>t_{f p}$.

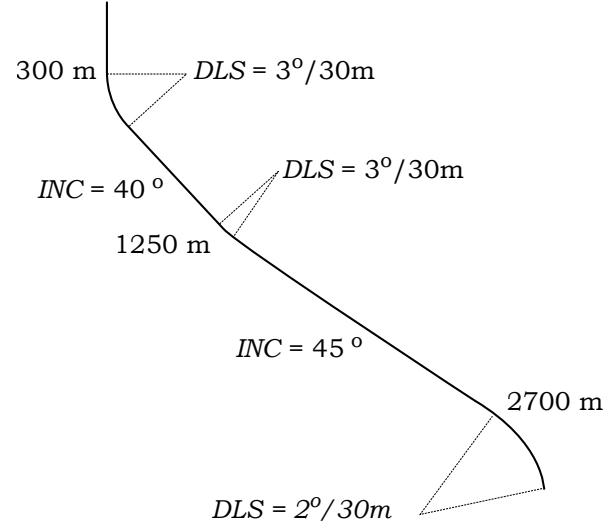

(a) Well A

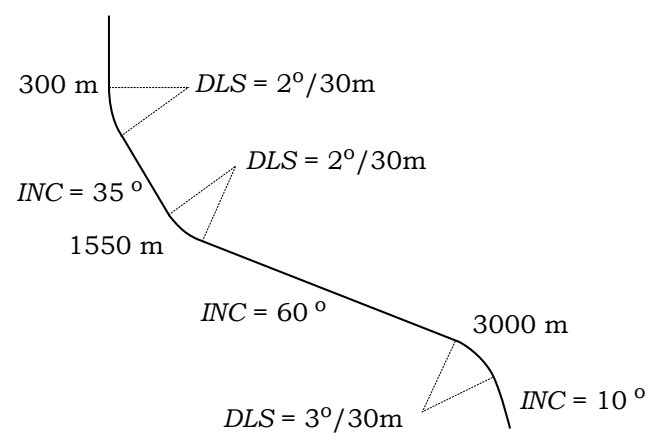

(b) Well B

Figure 5: Wellbore surveys of the two field wells

\section{Model comparison with field data}

To validate the modeling approach taken in the present work, a simulation study was undertaken to compare the behavior of the model to that of recorded field data. Two field comparisons are presented, with one exhibiting inerita dominated oscillations and the other exhibiting stick dominated oscillations, as categorized in [20].

\subsection{Simulation study case description}

Field data for two deviated wells, the surveys of which are shown in Figures 5a and 5b, are considered. Rotational data - rotary rpm and torque - is recorded at $100 \mathrm{~Hz}$ and provides a clear picture of the rpm, both set-points and realized values, used and during the drilling process and the torsional drill-string response.

The torque from the variable frequency drive is controlled with a PI controller with known gains of $k_{p}=38$ $\mathrm{kNm}$ per $\mathrm{m} / \mathrm{s}$ and $k_{i}=100 \mathrm{kNm}$ per $\mathrm{m} / \mathrm{s}^{2}$, see (10)-(13). In all cases presented herein, the drill-string starts at rest with zero torque at the surface. However, the stored torsional energy within the drill-string is not known. In both cases, the drill-string design is a simple directional assembly which is simplified to a 230 meter 53/4" OD BHA and monodiameter drillpipe to the surface. Downhole rpm and vibration data was collected for drilling performance improvement and control system verification and included 

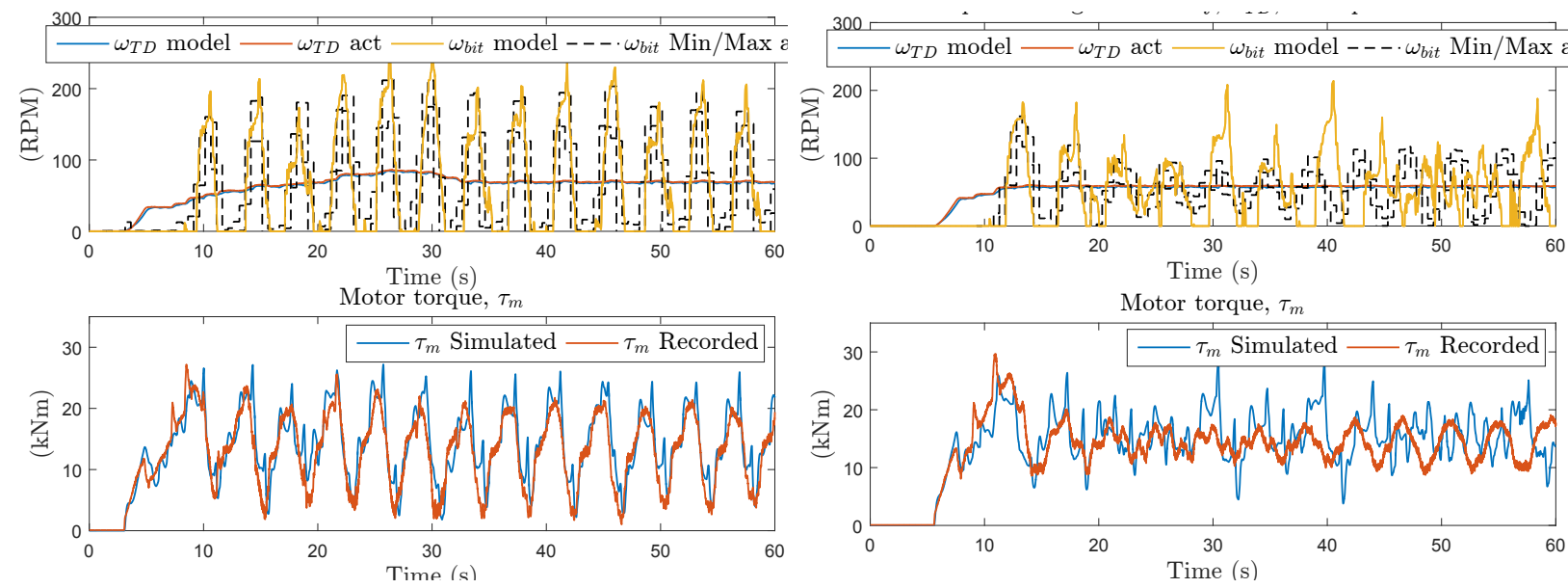

Figure 6: Left: Recorded and simulated drill-string response at a bit depth of 1,733 $\mathrm{m}$ in Well A, using fitting parameters: $\mu=0.34, f_{\text {rat }}=0.55, \omega_{c}=19(\mathrm{RPM})$. Right: Recorded and simulated drill-string response at a bit depth of 2,235 $\mathrm{m}$ in Well A, using fitting parameters: $\mu=0.35, f_{\text {rat }}=0.45, \omega_{c}=16(\mathrm{RPM})$.
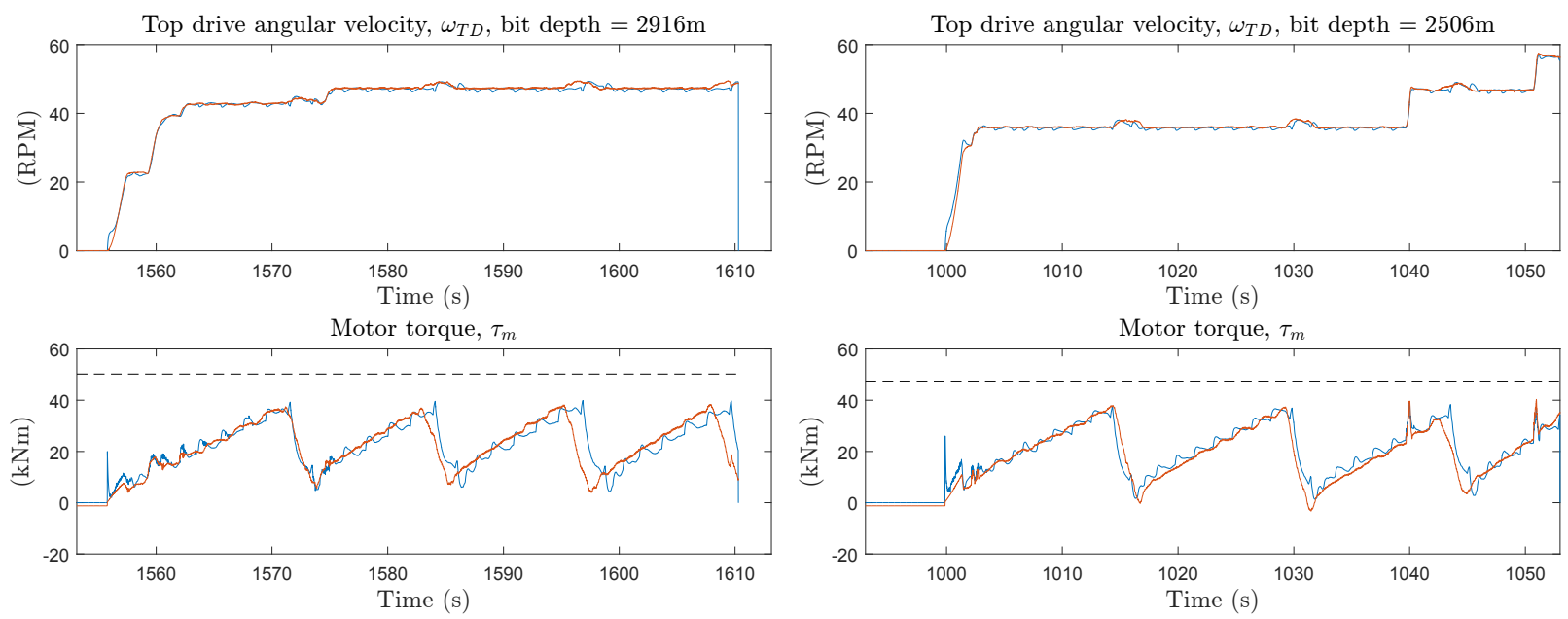

Figure 7: Left: Recorded and simulated drill-string response at a bit depth of 2,916 m in Well B. Right: Recorded and simulated drill-string response at a bit depth of 2,2506 $\mathrm{m}$ in Well B.

continuous low frequency $(0.5 \mathrm{~Hz})$ data as well as occasional burst sequences of high frequency data $(125 \mathrm{~Hz})$. For Well B, downhole data was sampled continuously at $125 \mathrm{~Hz}$ and then processed to record minimum, maximum and mean rpm for the 0.5 second sampling window.

\subsection{Well A: Inertia dominated oscillations}

Recorded field data and a model fit for two different depths, 1,733 $\mathrm{m}$ and 2,235 $\mathrm{m}$ are shown in Figures 6 and 6. In each figure, the top plot shows the surface (in red) and downhole (dashed) recorded data as well as the modeled data (in blue and yellow, respectively). The bottom plot shows surface torque, with recorded data in red and simulated data in blue. Only the Coulomb friction parameters changes between the two model fits, which is expected as the friction coefficient is expected to change as new rock is encountered and the side force is distribution on the drill string is changed.
In Figure 6 the max torque peak is repeated throughout, and the period is regular, indicating that the full max torque is reached in this stick slip limit cycle. This is not the case in Figure 6, however, as can be seen in the irregular max torques. The lower-amplitude oscillations that occur after this initial peak is due to the torque contribution from the static Coulomb friction at the upper-most kick-off point not being activated (see Fig. 5b), i.e. only the Coulomb friction in the lower section of the well contributes to the stick slip limit cycle, thus lowering the amplitude.

\subsection{Well B: Stick dominated oscillations}

The field data from Well $\mathbf{B}$ comes from a deeper section with a shallower profile - the tangent section of the well is $60^{\circ}$ instead of the $45^{\circ}$ as in Well $\mathbf{A}$, which results in a higher break-off torque. This, coupled with the fact that a lower RPM is used, causes Well B to exhibit stick dominated oscillations, where the majority of the limit cycle is 
spent building up the required break off torque. Downhole data was not available for this well, however, a fit of the surface data may still be made and is shown in Figure 7 and 7 . Here the torque build up can be clearly seen, which is comparatively slow relative to the torque release.

\subsection{Remarks on the model comparison}

The model fit is found to be very sensitive and the quality of fit varied from dataset to dataset. This is due to a number of factors:

- Pre-existing torsional stress state, the amount of twist existing in the drillstring, is not known at each connection event. Thus the total number of twists needed to break static friction is not known. This is very difficult to both model and quantify in field data since there is no method to to know the number of twists in the drillstring from the available recorded field data.

- The model only accounts for torsional state within the drillstring and does not take into account the axial stress state, hence once the drillstring begins moving axially, the model assumptions are violated and deviation between the field and simulated data appears.

\section{Conclusions and further work}

A distributed Coulomb friction model has been presented which is parameterized using three parameters: the static and dynamic friction, $F_{c}$ and $F_{d}$, or similarly, the coefficient of friction, $\mu$, and the ratio between static and dynamic friction, $f_{r a t}$, along the wellbore and the cutoff angular friction, $\omega_{c}$. The model is applied to two datasets and the surface and downhole behavior is found to match closely with model predictions in most cases. Furthermore, once axial motion is introduced, a key assumption of the presented model - that only torsional dynamics are considered - is violated.

\section{Acknowledgment}

This work was supported by the Research Council of Norway, ConocoPhillips, Det norske oljeselskap, Lundin, Statoil and Wintershall through the research center DrillWell (203525/O30) at IRIS and through the Schulich School of Engineering at the University of Calgary.

The authors also wish to acknowledge Shell International Exploration and Production for allowing use of their data and Sicco Dwars for his support of the effort. Finally, the authors wish to acknowledge Eric Cayeux at IRIS for helpful input to this work.

\section{References}

[1] S. Stokka, E. Cayeux, D. Gardner, S. Kragset, H.P. Lohne, E. Randeberg, H.J. Skadsem, B. Aas, H. Kyllingstad, T. Larsen et al., Long Reach Well
Concept, in IADC/SPE Drilling Conference and Exhibition (Forth Worth, 2016)

[2] R.J. Shor, M. Pryor, E. van Oort, Drillstring Vibration Observation, Modeling and Prevention in the Oil and Gas Industry, in ASME 2014 Dynamic Systems and Control Conference (2014)

[3] J.J. Bailey, I. Finnie, Transactions of the American Society of Mechanical Engineers pp. 122-127 (1960)

[4] D. Dashevskiy, J. Rudat, L. Pohle, Model-Based Stability Analysis of Torsional Drillstring Oscillations, in Proceedings of 2011 IEEE International Conferencce on Control Applications (Denver, CO, 2011), ISBN 9781457710636

[5] R.I. Leine, D.H. van Campen, W.J.G. Keultjes, Journal of Vibration and Acoustics 124, 209 (2002)

[6] K. Nandakumar, M. Wiercigroch, Journal of Sound and Vibration 332, 2575 (2013)

[7] X. Liu, N. Vlajic, X. Long, G. Meng, B. Balachandran, State-Dependent Delay Influenced Drill String Dynamics and Stability Analysis, in Volume 7B: 9th International Conference on Multibody Systems, Nonlinear Dynamics, and Control (ASME, 2013), p. V07BT10A065, ISBN 978-0-7918-5597-3

[8] J. Brett, SPE Drilling Engineering 7, 168 (1992)

[9] P.E. Pastusek, D. Ertas, L. Wang, J.R. Bailey, Proceedings of 2013 SPE / IADC Drilling Conference and Exhibition (2013)

[10] M. Kapitaniak, V. Vaziri, J. Páez, K. Nandakumar, M. Wiercigroch, International Journal of Mechanical Sciences 101-102, 324 (2015)

[11] T. Richard, C. Germay, E. Detournay, Comptes Rendus Mécanique 332, 619 (2004)

[12] T. Richard, C. Germay, E. Detournay, Journal of Sound and Vibration 305, 432 (2007)

[13] C. Germay, N. van de Wouw, H. Nijmeijer, R. Sepulchre, SIAM Journal on Applied Dynamical Systems 8, 527 (2009)

[14] J. Brett, A. Beckett, C. Holt, D. Smith, SPE Drilling Engineering 4, 223 (1989)

[15] G. Halsey, A. Kyllingstad, T. Aarrestad, D. Lysne, Drillstring Vibrations: Comparison Between Theory and Experiments on a Full-Scale Research Drilling Rig, in SPE/IADC Drilling Conference (Society of Petroleum Engineers, 1986), IADC/SPE 14760, pp. 311-321, ISBN 9781555636074

[16] D. Zhao, S. Hovda, S. Sangesland, Journal of Petroleum Science and Engineering In press (2016)

[17] U.J.F. Aarsnes, O.M. Aamo, Journal of Sound and Vibration 360, 239 (2016)

[18] U.J.F. Aarsnes, N. van de Wouw, in review (2017)

[19] C. Germay, V. Denoël, E. Detournay, Journal of Sound and Vibration 325, 362 (2009)

[20] U.J.F. Aarsnes, R.J. Shor, Journal of Petroleum Science and Engineering (accepted) (2017)

[21] M. Sheppard, C. Wick, T. Burgess, SPE Drilling Engineering 2, 344 (1987) 